\title{
Mediterranean Diet and Risk for Alzheimer's Disease
}

\author{
Nikolaos Scarmeas, MD,${ }^{1-3}$ Yaakov Stern, PhD, ${ }^{1-3}$ Ming-Xin Tang, PhD,${ }^{1,4}$ Richard Mayeux, MD,,${ }^{1-3}$ \\ and Jose A. Luchsinger, $\mathrm{MD}^{1,5}$
}

Objective: Previous research in Alzheimer's disease (AD) has focused on individual dietary components. There is converging evidence that composite dietary patterns such as the Mediterranean diet $(\mathrm{MeDi})$ is related to lower risk for cardiovascular disease, several forms of cancer, and overall mortality. We sought to investigate the association between $\mathrm{MeDi}$ and risk for AD.

Methods: A total of 2,258 community-based nondemented individuals in New York were prospectively evaluated every 1.5 years. Adherence to the $\mathrm{MeDi}$ (zero- to nine-point scale with higher scores indicating higher adherence) was the main predictor in models that were adjusted for cohort, age, sex, ethnicity, education, apolipoprotein E genotype, caloric intake, smoking, medical comorbidity index, and body mass index.

Results: There were 262 incident AD cases during the course of 4 ( \pm 3.0 ; range, 0.2-13.9) years of follow-up. Higher adherence to the $\mathrm{MeDi}$ was associated with lower risk for $\mathrm{AD}$ (hazard ratio, $0.91 ; 95 \%$ confidence interval, $0.83-0.98 ; p=0.015$ ). Compared with subjects in the lowest MeDi tertile, subjects in the middle MeDi tertile had a hazard ratio of $0.85(95 \%$ confidence interval, $0.63-1.16)$ and those at the highest tertile had a hazard ratio of 0.60 (95\% confidence interval, $0.42-0.87)$ for $\mathrm{AD}(p$ for trend $=0.007$ ).

Interpretation: We conclude that higher adherence to the $\mathrm{MeDi}$ is associated with a reduction in risk for $\mathrm{AD}$.

Ann Neurol 2006;59:912-921

Diet may play an important role in the causation and prevention of $\mathrm{AD} .^{1,2}$ Dietary restriction in animals extends their life $\operatorname{span}^{3}$ and increases the resistance of neurons to degeneration. ${ }^{3}$ However, epidemiological data on diet and $\mathrm{AD}$ have been conflicting. Higher intake of vitamin $\mathrm{C},{ }^{4-6}$ vitamin $\mathrm{E},{ }^{4-8}$ flavonoids, ${ }^{9}$ unsaturated fatty acids, ${ }^{10-12}$ fish $^{12,13}$; higher levels of vitamin $\mathrm{B} 12^{14-16}$ and folate ${ }^{15,16}$; modest to moderate ethanol $^{17-21}$; and lower total fats ${ }^{22,23}$ have been related to a lower risk for $\mathrm{AD}$ or slower cognitive decline. At the same time, other studies have found that the risk for $\mathrm{AD}$ or cognitive decline is not associated to intake of antioxidants, such as vitamin $\mathrm{C},{ }^{24}$ vitamin $\mathrm{E},{ }^{24}$ and carotenes, ${ }^{24}$ fats, ${ }^{25}$ or levels of vitamin B12. ${ }^{26}$

There is paucity of data regarding the effect of composite dietary patterns (rather than individual foods or nutrients) on the risk for $\mathrm{AD}$. Dietary pattern analysis in relation to many other diseases (ie, cirrhosis or various cancers) has recently received growing attention because individuals do not consume foods or nutrients in isolation, but rather as components of their daily diet. Defining diet by dietary patterns has the ability to capture its multidimensionality whereas reducing its apparent complexity because patterns can integrate complex or subtle interactive effects of many dietary constituents and bypass problems generated by multiple testing and the high correlations that may exist among these constituents. ${ }^{27}$ Dietary patterns can be developed a priori from previous knowledge concerning a favorable or adverse health effect of various dietary constituents.

One such dietary pattern is the Mediterranean diet $(\mathrm{MeDi})$, which has received increased attention in recent years because of converging ecological, ${ }^{28}$ analytical-observational, ${ }^{29-33}$ and interventional ${ }^{34,35}$ evidence relating it to lower risk for cardiovascular disease, several forms of cancer, and overall mortality. The $\mathrm{MeDi}$ is characterized by high intake of vegetables, legumes, fruits, and cereals; high intake of unsaturated fatty acids (mostly in the form of olive oil), but low intake of saturated fatty acids; a moderately high intake of fish; a low-to-moderate intake of dairy products (mostly cheese or yogurt); a low intake of meat and poultry; and a regular but moderate amount of ethanol, primarily in the form of wine and generally during meals. ${ }^{31}$ Therefore, the MeDi appears to include many of the
From the ${ }^{1}$ Taub Institute for Research in Alzheimer's Disease and the Aging Brain; ${ }^{2}$ Gertrude H. Sergievsky Center; ${ }^{3}$ Department of Neurology; ${ }^{4}$ Division of Biostatistics, Joseph P. Mailman School of Public Health; and ${ }^{5}$ Department of Medicine, Columbia University, New York, NY.

Received Dec 22, 2005, and in revised form Mar 3, 2006. Accepted for publication Mar 10, 2006.
Published online Apr 18, 2006, in Wiley InterScience (www.interscience.wiley.com). DOI: 10.1002/ana.20854

Address correspondence to Dr Scarmeas, Columbia University Medical Center, 622 West 168th Street; PH 19th Floor; New York, NY 10032. E-mail: ns257@columbia.edu 
components reported as potentially beneficial for $\mathrm{AD}$ and cognitive performance.

We examined the association between $\mathrm{MeDi}$ and $\mathrm{AD}$ using data from the Washington Heights-Inwood Columbia Aging Project (WHICAP). We hypothesized that higher adherence to the MeDi would be associated with lower risk for $\mathrm{AD}$.

\section{Subjects and Methods}

Sample and Procedures

This study included participants of two related cohorts recruited in 1992 (WHICAP 1992) and 1999 (WHICAP 1999), which were identified (via ethnicity and age stratification processes) from a probability sample of Medicare beneficiaries residing in an area of three contiguous census tracts within a geographically defined area of northern Manhat$\tan .23,24,36,37$ The same assessments and study procedures were used in both cohorts. At entry, a physician elicited each subject's medical and neurological history and conducted a standardized physical and neurological examination. All available ancillary information (medical charts, computed tomography scans, or magnetic resonance images) was considered in the evaluation. A global summary score on the Clinical Dementia Rating $(\mathrm{CDR})^{38}$ scale also was assigned. Each subject also underwent a structured in-person interview including an assessment of health and function and a neuropsychological battery. ${ }^{39}$ The neuropsychological battery contained tests of memory (short- and long-term verbal and nonverbal), orientation, abstract reasoning (verbal and nonverbal); language (naming, verbal fluency, comprehension, and repetition), and construction (copying and matching).

A consensus diagnosis for the presence or absence of dementia was made at a diagnostic conference of neurologists and neuropsychologists where information of all the above evaluations was presented. Evidence of cognitive deficit (based on the neuropsychological scores as described above), evidence of impairment in social or occupational function (as assessed by the Blessed Dementia Rating Scale, the Schwab and England Activities of Daily Living Scale, and the physician's assessment), and evidence of cognitive and socialoccupational function decline compared with the past were the criteria used for the diagnosis of dementia as required by the Diagnostic and Statistical Manual of Mental Disorders, Revised Third Edition (DSM-III-R). The type of dementia was subsequently determined. For the diagnosis of probable or possible $\mathrm{AD}$, the criteria of the National Institute of Neurological and Communicative Disorders and StrokeAlzheimer's Disease and Related Disorders Association ${ }^{40}$ were used. Because in these criteria stroke does not preclude the diagnosis of $\mathrm{AD}$ (unless cerebrovascular disease was considered the primary cause of the dementia), the diagnosis of $\mathrm{AD}$ with concomitant stroke also was assigned. Dietary data were not available to the consensus panel and were not considered in the diagnostic process.

Subjects were followed up at intervals of approximately 1.5 years, repeating the baseline examination and consensus diagnosis at each follow-up. The initial sample for this study was 4,166 individuals, of whom 3,436 were without dementia at initial evaluation (Fig 1). Because the dietary assess-

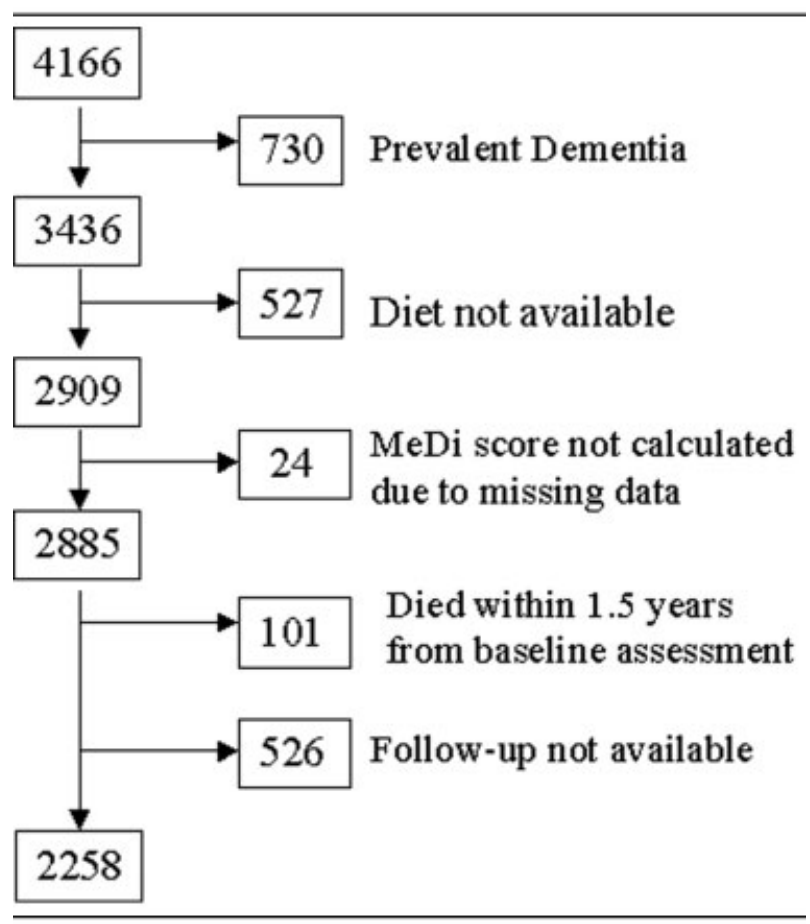

Fig 1. Flow chart describing sample size. MeDi $=$ Mediterranean diet.

ment component was added after initiation of the study, dietary information was missing for some subjects $(\mathrm{n}=527)$. An additional 24 subjects were excluded due to incomplete dietary information. From the remaining 2,885 subjects, follow-up was not available for 627 (including 101 because they died relatively soon after the baseline assessment). Thus, the initial analytic sample for the survival analyses comprised 2,258 subjects (1,037 [46\%] from the WHICAP 1992 and 1,221 [54\%] from the WHICAP 1999 cohort).

\section{Evaluation}

\section{PREDICTORS}

Diet. Dietary data regarding average food consumption over the past year were obtained using a 61-item version of Willett's semiquantitative food frequency questionnaire (SFFQ; Channing Laboratory, Cambridge, MA). ${ }^{41}$ Trained interviewers administered the SFFQ in English or Spanish. We have previously reported validity (using two 7-day food records) and reliability (using two 3-month frequency assessments) of various components of the SFFQ in WHICAP. $23,24,36$

We followed the method that Trichopoulou and colleagues $^{31}$ described previously for the construction of the $\mathrm{MeDi}$ score. More specifically, we first regressed caloric intake (measured in kilocalories) and calculated the derived residuals of daily gram intake (as Willet and Stampfer ${ }^{42}$ recommended) for each of the following seven categories (which define the components of the $\mathrm{MeDi}$ that Trichopoulou and colleagues $^{31}$ defined previously): dairy, meat, fruits, vegetables, legumes, cereals, and fish. A value of 0 or 1 was assigned to each of these seven groups, using sex-specific medians as cutoffs. For beneficial components (fruits, 
vegetables, legumes, cereals, and fish), individuals whose consumption was below the median were assigned a value of 0 , and individuals whose consumption was at or above the median were assigned a value of 1 . For components presumed to be detrimental (meat and dairy products), individuals whose consumption was below the median were assigned a value of 1 , and individuals whose consumption was at or above the median were assigned a value of 0 . For fat intake (the eighth food category), we used the ratio of daily consumption (in grams) of monounsaturated lipids to saturated lipids ${ }^{31}$ (again using sex-specific median cutoffs for assignment values of 0 for low and 1 for high). For alcohol intake (the ninth food category), subjects were assigned a score of 0 for either no $(0 \mathrm{gm} /$ day $)$ or more than moderate $(\geq 30 \mathrm{gm} /$ day) consumption, and a value of 1 for mild-moderate alcohol consumption ( $>0$ to $<30 \mathrm{gm} /$ day). This agrees with Trichopoulou's $^{31}$ previous reports that consider moderate amount of alcohol consumption as another characteristic component of the MeDi. We classified alcohol consumption dichotomously, because of the skewed distribution of alcohol in our population (68\% reporting no alcohol intake, 31\% reporting less than $30 \mathrm{gm} /$ day [mild-to-moderate intake], and $1 \%$ reporting $\geq 30 \mathrm{gm} /$ day [heavy intake]). The MeDi score was generated for each participant by adding the scores in the food categories (theoretically ranging from 0-9) with the higher score indicating stricter adherence to the MeDi. Median intake of consumption for $\mathrm{MeDi}$ food categories is presented in Table 1 . We used MeDi score from the baseline visit as the main predictor in the survival analyses.

Covariates. Age (years), education (years), caloric intake (kcal), and body mass index (weight in kilograms divided by height in square meters $\left.\left[\mathrm{kg} / \mathrm{m}^{2}\right]\right)^{43}$ were used as continuous variables. We also considered cohort (1992 cohort as reference), sex (men as reference), and smoking status at baseline evaluation (no smoking as reference). Ethnic group was based on self-report using the format of the 1990 census. ${ }^{44}$ Participants then were assigned to one of four groups: Black (non-Hispanic), Hispanic, White (non-Hispanic), or Other. Ethnicity was used as a dummy variable with White (nonHispanic) as the reference. Apolipoprotein E (APOE) genotype was used dichotomously: absence of $\varepsilon 4$ allele versus presence of either 1 or $2 \varepsilon 4$ alleles.
A modified version ${ }^{45,46}$ of the Charlson Index of Comorbidity ${ }^{47}$ (referred to as "comorbidity index") was included as a continuous variable. It included items for myocardial infarct, congestive heart failure, peripheral vascular disease, hypertension, chronic obstructive pulmonary disease, arthritis, gastrointestinal disease, mild liver disease, diabetes, chronic renal disease, and systemic malignancy from the baseline visit. All items received weights of one, with the exception of chronic renal disease and systemic malignancy, which were weighted two.

STATISTICAL ANALYSES. Baseline characteristics of patients by availability of follow-up, outcome of interest, and $\mathrm{MeDi}$ tertiles were compared using $t$ test or analysis of variance for continuous variables and $\chi^{2}$ test for categorical variables.

Mediterranean Diet Score Stability. We used generalized estimating equations (GEEs) to test whether there were significant changes of MeDi score over time for a subset of subjects with more than one dietary assessment. These repeated dietary assessments were performed to obtain information on the temporal stability of dietary reporting. GEE takes into account the multiple visits per subject and that the characteristics of the same individual over time are likely to be correlated. The repeated measures for each subject are treated as a cluster. The GEE model included the MeDi score as the dependent variable and time (years) as predictor. A significant time effect would indicate a significant change of $\mathrm{MeDi}$ score over time. We performed separate exploratory analyses for subjects who experienced development of $\mathrm{AD}$ and for ones who remained nondemented.

Survival Analyses. We calculated Cox proportional hazards models with $\mathrm{AD}$ as the dichotomous outcome. Subjects who experienced development of other dementias $(n=32)$ were excluded from these analyses. In exploratory analyses that censored (rather than excluded) these subjects, the results were unchanged (these analyses are not presented). The timeto-event variable was time from recording of baseline diet to first visit of dementia diagnosis; subjects who did not experience development of dementia were censored at the time of their last follow-up. The main predictor was MeDi score (from the baseline visit) as a continuous variable initially and

Table 1. Median Daily Intake for Individual Food Categories by Mediterranean Diet Score Tertiles and Overall

\begin{tabular}{lcccc}
\hline Food Categories & $\begin{array}{c}\text { Low Tertile } \\
\text { (MeDi score 0-3) }\end{array}$ & $\begin{array}{c}\text { Middle Tertile } \\
\text { (MeDi score 4-5) }\end{array}$ & $\begin{array}{c}\text { High Tertile } \\
\text { (MeDi score 6-9) }\end{array}$ & $\begin{array}{c}\text { All (25th, 75th } \\
\text { percentiles) }\end{array}$ \\
\hline Dairy, gm/day & 246 & 174 & 151 & $182(128-292)$ \\
Meat, gm/day & 101 & 86 & 65 & $85(60-119)$ \\
Vegetable, gm/day & 165 & 202 & 243 & $197(153-250)$ \\
Fruit, gm/day & 406 & 471 & 556 & $472(372-582)$ \\
Legumes, gm/day & 44 & 58 & 78 & $57(38-90)$ \\
Cereal, gm/day & 155 & 186 & 215 & $184(140-233)$ \\
Fish, gm/day & 15 & 21 & 47 & $20(14-47)$ \\
MUFA/SFA ratio & 0.57 & 0.82 & 0.97 & $0.80(0.18-1.37)$ \\
Mild-to-moderate ethanol, \% & 21 & 33 & 45 & 32
\end{tabular}

Values represent calorie-adjusted residuals. For presentation purposes, a constant representing the predicted value for each category for $2,500 \mathrm{kcal}$ for men and 2,000kcal for women has been added. ${ }^{42} \mathrm{MeDi}=$ Mediterranean diet; MUFA $=$ monounsaturated fatty acids; SFA $=$ saturated fatty acids. 
in tertiles form subsequently (used for trend test calculation). We simultaneously adjusted for the following variables: cohort, age at intake in the study, sex, ethnicity, education, APOE, smoking, comorbidity index, and body mass index. Although caloric intake adjusted residuals were used in the MeDi score calculation, we also included caloric intake as a covariate in the models (as Willet and colleagues ${ }^{42}$ recommended). All predictors were used as time-constant covariates. We performed two additional sets of sensitivity analyses. First, we recalculated the models using as dementia diagnosis date the last date when subjects were considered to be cognitively nondemented. Second, we recalculated the models using as dementia date the midpoint between last nondementia diagnosis and first dementia diagnosis.

In other exploratory models, we repeated the analyses excluding subjects who were diagnosed as AD with stroke (ie, using only $\mathrm{AD}$ without stroke as the outcome). To increase our confidence that the degree of adherence to MeDi was not affected by early, subclinical dementia process, we performed additional analyses. First, we recomputed the Cox models excluding subjects with mild cognitive deficits at baseline (ie, CDR $=0.5$ ). Second, we excluded both subjects with mild cognitive deficits at baseline (ie, CDR $=0.5$ ) and subjects who were followed for less than 2 years.

To examine whether possible associations between MeDi and risk for $\mathrm{AD}$ were driven by associations of particular food categories, in further supplementary analyses, we included all nine individual components used to calculate the $\mathrm{MeDi}$ score in stepwise forward selection Cox models (entry criterion: $p<0.05$; removal criterion: $p>0.05$ ). The Cox models fulfilled the proportionality assumption (martingale residuals method).

RATES OF COGNITIVE DECLINE IN RELATION TO THE MEDITERRANEAN DIET. The primary outcome in these analyses was rate of decline in cognition as assessed at each study visit. Using 12 neuropsychological tests from the administered battery, ${ }^{48-54}$ we calculated a composite cognitive measure. Details about this procedure have been published previously. ${ }^{55}$ In brief, to derive the composite measure, we first transformed each of the 12 individual cognitive tests into $Z$-scores. Means and standard deviations for each test were calculated from baseline scores of nondemented subjects, and $Z$-scores were averaged initially in cognitive domains, which were subsequently averaged again to produce the composite cognitive score. The $Z$-score of the composite cognitive measure at each evaluation was the primary outcome.

We used GEEs to test whether MeDi adherence was associated with differential rates of cognitive change. The repeated cognitive performance scores for each subject were treated as a cluster. The GEE models included the composite cognitive measure as the dependent variable and, as predictors, cohort, age, sex, education, ethnicity, baseline cognitive performance, $\mathrm{MeDi}$ at baseline, time (in years from baseline assessment), and a MeDi $\times$ time interaction. A significant interaction term would indicate differential rates of change in cognitive function as a function of $\mathrm{MeDi}$ adherence.

\section{Results}

\section{Missing Data Analyses}

Compared with subjects with available dietary information, subjects with missing dietary information ( $\mathrm{n}=$ 527) (see Fig 1) had slightly lower education (9.1 vs 9.9 years; $p=0.001$ ). Subjects with missing dietary information also had higher proportions of dementia (17.5 vs $11 \% ; p<0.001)$ and higher mortality (32 vs $18 \% ; p<0.001)$, which may be related to that the dietary assessment was added after initiation of the study and was not available for subjects recruited earlier on. There were no significant differences between subjects with missing and those with available dietary information in age ( 76.7 vs $77 ; p=0.30)$, sex $(33 \%$ male vs 33\% male; $p=0.68$ ), ethnicity (White 25\%, Black 31\%, Hispanic 43\%, Other $1 \%$ vs White 27\%, Black 33\%, Hispanic 39\%, Other 1\%; $p=0.24)$, medical comorbidity index (2.1 vs $2.0 ; p=0.27$ ), or APOE genotype ( $\varepsilon 4$ carriers 27 vs $28 \% ; p=0.79$ ).

Compared with subjects with available cognitive follow-up ( $\mathrm{n}=2,258$ ), subjects with missing follow-up ( $\mathrm{n}=627$ ) (see Fig 1 ) were slightly younger (76.4 vs 77.2 years; $p=0.01$ ), had lower education (9.4 vs 10.0 years; $p=0.005)$, more medical comorbidities (2.2 vs $1.9 ; p=0.002$ ), and higher mortality (30 vs $15 \% ; p<0.001)$. There were no significant differences between subjects with missing and those with available follow-up in caloric intake $(1,466$ vs 1,$428 ; p=0.12)$, sex $(35 \%$ male vs $33 \%$ male; $p=0.42)$, ethnicity (White 26\%, Black 36\%, Hispanic 38\%, Other 1\% vs White 28\%, Black 33\%, Hispanic 38\%, Other 2\%; $p=0.39$ ), or APOE genotype ( $\varepsilon 4$ carriers 29 vs $28 \%$; $p=0.67)$. Most important, there was no difference in MeDi score ( 4.3 vs $4.4 ; p=0.21$ ).

Examining evaluations for subjects who had available follow-up, 76 subjects were missing 1 evaluation, 11 were missing 1 evaluation, and 2 were missing 3 evaluations. Examining, in particular, the time period either between the dementia incidence evaluation and the evaluation preceding that (for $\mathrm{AD}$ subjects) or between the last evaluation and the evaluation preceding that (for nondemented subjects), only 23 subjects (7 $\mathrm{AD}$ and 16 nondemented) had 1 missing evaluation and only 2 subjects ( $1 \mathrm{AD}$ and 1 nondemented) had 2 missing evaluations. Therefore, missing evaluations for subjects with available follow-up was a rare phenomenon in our study.

\section{Stability of Mediterranean Diet Score}

There were 390 subjects with multiple dietary assessments who did not experience development of $\mathrm{AD}$ or other dementia during follow-up. There were 2 dietary assessments available for 308 subjects, 3 for 71 subjects, and 4 for 11 subjects. The mean time interval between dietary assessments was 7.1 years (standard de- 
Table 2. Demographic, Clinical, and Dietary Characteristics

\begin{tabular}{|c|c|c|c|c|}
\hline Characteristics & $\begin{array}{l}\text { Nondemented } \\
(\mathrm{n}=1,964)\end{array}$ & $\begin{array}{c}\text { Incident AD } \\
(\mathrm{n}=262)\end{array}$ & $\begin{array}{c}\text { All } \\
(\mathrm{N}=2,226)\end{array}$ & $p$ \\
\hline Mean age, yr (SD) & $76.5(6.3)$ & $81.8(6.9)$ & $77.2(6.6)$ & $<0.001$ \\
\hline Male sex, n (\%) & $634(32)$ & $86(33)$ & $720(32)$ & 0.86 \\
\hline Mean education, yr (SD) & $10.5(4.6)$ & $7.0(4.5)$ & $10.1(4.7)$ & $<0.001$ \\
\hline Ethnicity, n (\%) & & & & $<0.001$ \\
\hline White & $592(30)$ & $28(11)$ & $620(28)$ & \\
\hline Black & $636(32)$ & $86(33)$ & $722(32)$ & \\
\hline Hispanic & $705(36)$ & $143(55)$ & $848(38)$ & \\
\hline Other & $31(2)$ & $5(2)$ & $36(2)$ & \\
\hline Presence of $\varepsilon 4$ allele, n (\%) & $452(27)$ & $74(32)$ & $526(27)$ & 0.10 \\
\hline Smoking, n (\%) & $242(12)$ & $33(13)$ & $275(12)$ & 0.90 \\
\hline Mean comorbidity index (SD) & $1.9(1.4)$ & $1.9(1.5)$ & $1.9(1.4)$ & 0.87 \\
\hline Mean energy, kcal (SD) & $1422(513)$ & $1475(560)$ & $1428(519)$ & 0.15 \\
\hline Mean body mass index (SD) & $27.5(5.5)$ & $26.5(5.9)$ & $27.4(5.5)$ & 0.005 \\
\hline Mean MeDi score (SD) & $4.4(1.7)$ & $4.2(1.7)$ & $4.3(1.7)$ & 0.05 \\
\hline Low MeDi tertile, n (\%) & $326(32)$ & $96(37)$ & $722(32)$ & 0.23 \\
\hline Middle MeDi tertile, n (\%) & $823(42)$ & $107(41)$ & $930(42)$ & \\
\hline High MeDi tertile, n (\%) & $515(26)$ & $59(23)$ & $574(26)$ & \\
\hline
\end{tabular}

The sum of nondemented and incident Alzheimer's disease (AD) is smaller than all subjects combined (ie, $\mathrm{n}=2$,258; see Fig 1 ) because 32 subjects who experienced development of dementia other than $\mathrm{AD}$ are not included in this table. MeDi $=$ Mediterranean diet; $\mathrm{SD}=$ standard deviation.

viation, 2.36; range, 1-12.8). The reported $\mathrm{MeDi}$ score was stable $(\beta=-0.01 ; p=0.41)$.

There were 89 subjects with multiple dietary assessments who developed AD during follow-up; there were 2 assessments available for 78 participants, 3 for 8 participants, and 4 for 3 participants. The mean time interval between dietary assessments was 8.1 years (standard deviation, 1.9; range, 1.8-11.9). Again, the reported MeDi score did not change over time $(\beta=$ $-0.05 ; p=0.09$ ).

\section{Clinical-Demographic-Dietary Characteristics}

Subjects were followed (until AD incidence or last follow-up for subjects who remained without demented) for an average of 4.0 ( \pm 3.0 ; range, $0.2-13.9)$ years. Overall, 294 subjects experienced development of incident dementia; AD developed in 262 of these subjects (184/262 had AD without stroke).

Compared with subjects who remained without dementia, subjects in whom AD had developed at follow-up were older, less educated, and had a lower body mass index (Table 2). There was a higher proportion of Hispanics and a lower proportion of Whites (non-Hispanic) among subjects who experienced development of $\mathrm{AD}$. Presence of $\varepsilon 4$ allele was more frequent and caloric intake was higher for subjects in whom AD developed compared with subjects who remained without dementia, but the associations were not significant. Subjects who did and did not acquire AD did not differ in sex, medical comorbidity index, or smoking status. Compared with subjects who remained nondemented, subjects who experienced development of $\mathrm{AD}$ had lower MeDi scores.
There was no association between MeDi score and age, sex, education, APOE genotype, medical comorbidity index, or body mass index (Table 3 ). Hispanics adhered more and Blacks less to the MeDi pattern. Subjects adhering more to the $\mathrm{MeDi}$ tended to smoke less and had lower caloric intake.

\section{Mediterranean Diet and Risk for Alzheimer's Disease and Cognitive Decline}

Higher adherence to the MeDi was associated with significantly lower risk for development of AD (Table 4 and Fig 2). The results were similar in adjusted and unadjusted models. Each additional unit of the MeDi score was associated with 9 to $10 \%$ less risk for development of $\mathrm{AD}$. Compared with subjects in the lowest MeDi tertile (low adherence to the MeDi), subjects in the middle MeDi score tertile had 15 to $21 \%$ less risk for development of $\mathrm{AD}$, whereas those at the highest tertile (high adherence to the $\mathrm{MeDi}$ ) had 39 to $40 \%$ less risk for development of $\mathrm{AD}$, with a significant trend for a dose-response effect. In sensitivity analyses using alternative dementia diagnosis dates (either last date when subjects were deemed nondemented or the midpoint between last nondementia and first dementia diagnosis) the associations were unchanged.

The coefficients remained virtually unchanged and the associations significant in models excluding subjects with baseline evidence of mild cognitive impairment $(\operatorname{CDR} p=0.5): 1,898$ subjects at risk with 156 incident $\mathrm{AD}$ cases; hazard ratio, 0.88 (95\% confidence interval, $0.80-0.97) ; p=0.007$, tertile analyses; $p$ for trend $=0.018$. When both persons with $\mathrm{CDR}=0.5$ 
Table 3. Demographic and Clinical Characteristics by Mediterranean Diet Score Tertiles

\begin{tabular}{|c|c|c|c|c|}
\hline Characteristics & $\begin{array}{c}\text { Low Tertile } \\
\text { (MeDi score 0-3) }\end{array}$ & $\begin{array}{l}\text { Middle Tertile } \\
\text { (MeDi score 4-5) }\end{array}$ & $\begin{array}{c}\text { High Tertile } \\
\text { (MeDi score 6-9) }\end{array}$ & $p$ \\
\hline WHICAP 1999 cohort, N (\%) & $395(55)$ & $508(55)$ & $312(54)$ & 0.99 \\
\hline Mean age, yr (SD) & $77.3(6.8)$ & $77.0(6.6)$ & $77.3(6.3)$ & 0.50 \\
\hline Male sex, n (\%) & $218(30)$ & $303(33)$ & $199(35)$ & 0.23 \\
\hline Mean education, yr (SD) & $10.2(4.5)$ & $9.9(4.8)$ & $10.1(4.9)$ & 0.45 \\
\hline Ethnicity, n (\%) & & & & $<0.001$ \\
\hline White & $204(28)$ & $251(27)$ & $165(29)$ & \\
\hline Black & $280(39)$ & $292(31)$ & $150(26)$ & \\
\hline Hispanic & $225(31)$ & $374(40)$ & $249(43)$ & \\
\hline Other & $13(2)$ & $13(1)$ & $10(2)$ & \\
\hline Presence of $\varepsilon 4$ allele, n (\%) & $164(27)$ & $214(27)$ & $148(29)$ & 0.68 \\
\hline Smoking, n (\%) & $111(15)$ & $119(13)$ & $45(8)$ & $<0.001$ \\
\hline Mean comorbidity index (SD) & $1.9(1.4)$ & $1.9(1.4)$ & $2.0(1.5)$ & 0.95 \\
\hline Mean energy, kcal (SD) & $1,498(605)$ & $1,399(467)$ & $1,387(473)$ & $<0.001$ \\
\hline Body mass index (SD) & $27.5(5.8)$ & $27.5(5.5)$ & $27.2(5.3)$ & 0.55 \\
\hline
\end{tabular}

WHICAP $=$ Washington Heights-Inwood Columbia Aging Project; MeDi = Mediterranean diet; SD = standard deviation.

and those followed for less than 2 years were excluded, the coefficients remained essentially unchanged: 1,575 subjects at risk with 134 incident $\mathrm{AD}$ cases; hazard ratio: 0.89 (95\% confidence interval, $0.80-0.98) ; p=$ 0.020 , tertile analyses; $p$ for trend $=0.027$. Results were similar in adjusted models.

When the same models were run with probable $\mathrm{AD}$ without stroke as the outcome (excluding AD with coexisting stroke, $\mathrm{n}=78$ ), the associations were unchanged: 2,144 subjects at risk with 184 incident probable AD without stroke; hazard ratio, $0.90 \quad(95 \%$ confidence interval, 0.83-0.98); $p=0.015$, tertile analyses; $p$ for trend $=0.018$. Adjusted models produced similar results.

Regarding associations of other nutrition-related elements with risk for $\mathrm{AD}$, and in agreement with previous publications, ${ }^{23,56}$ lower caloric intake $(0.9997$ for each additional kilocalorie; range, $0.9994-0.9999 ; p=$
$0.007)$ and higher body mass index (0.96; range, $0.93-$ $0.98 ; p=0.002)$ were related to lower risk for $\mathrm{AD}$ in the adjusted models.

In unadjusted Cox models that included the nine individual dietary components of the $\mathrm{MeDi}$ as predictors, mild-to-moderate alcohol consumption (0.61 $[0.45-0.82] ; p=0.001)$ and higher vegetable consumption (0.76 [0.60-0.97]; $p=0.030$ ) were associated with decreased risk for AD. In adjusted models, none of the individual components was a significant $\mathrm{AD}$ predictor.

In GEE analyses, there was a significant $\mathrm{MeDi} \times$ time interaction $\beta=0.003 ; p=0.047$ ) indicating an association between higher adherence to $\mathrm{MeDi}$ and slower cognitive decline: each additional unit of adherence to the MeDi was associated with $0.3 \%$ of a standard deviation less decline per year.

Table 4. Cox Proportional Hazard Ratios for Alzheimer's Disease by Mediterranean Diet Score Either in Continuous or in Tertile Form

\begin{tabular}{|c|c|c|c|c|c|c|}
\hline \multirow[b]{2}{*}{ Model } & \multirow{2}{*}{$\begin{array}{c}\text { At } \\
\text { Risk, } n\end{array}$} & \multicolumn{3}{|c|}{ MeDi Continuous } & \multicolumn{2}{|c|}{ MeDi Tertiles } \\
\hline & & $\mathrm{AD}(\%)$ & HR (95\% CI) & $p$ & HR (95\% CI) & $p$ for Trend \\
\hline $1^{\mathrm{a}}$ & 2,226 & $262(12)$ & $0.90(0.83-0.96)$ & 0.003 & & 0.003 \\
\hline Low & & & & & 1 (reference) & \\
\hline Middle & & & & & $0.79(0.60-1.04)$ & \\
\hline High & & & & & $0.61(0.44-0.85)$ & \\
\hline $2^{b}$ & 1,759 & $219(12)$ & $0.91(0.83-0.98)$ & 0.015 & & 0.007 \\
\hline Low & & & & & 1 (reference) & \\
\hline Middle & & & & & $0.85(0.63-1.16)$ & \\
\hline High & & & & & $0.60(0.42-0.87)$ & \\
\hline
\end{tabular}

The initial number at risk $(\mathrm{n}=2226)$ is smaller than all subjects combined (ie, $\mathrm{n}=2258$; see Fig 1$)$ because of excluding 32 subjects who experienced development of dementia other than Alzheimer's disease (AD).

${ }^{\text {a }}$ Model 1 is unadjusted.

${ }^{\mathrm{b}}$ Model 2 is adjusted for cohort, age, sex, ethnicity, education, apolipoprotein E genotype, caloric intake, smoking, comorbidity index, and body mass index.

$\mathrm{MeDi}=$ Mediterranean diet; $\mathrm{HR}=$ hazard ratio; $\mathrm{CI}=$ confidence interval. 


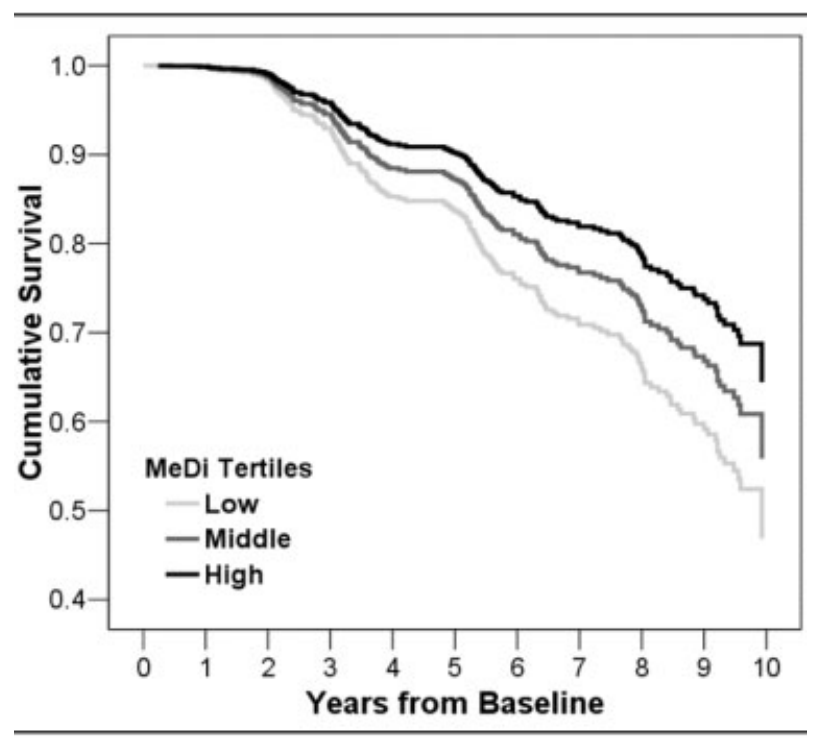

Fig 2. Survival curves based on Cox analysis comparing cumulative Alzheimer's disease $(A D)$ incidence in subjects belonging to each Mediterranean diet (MeDi) tertile ( $\mathrm{p}$ for trend $=0.007$ ). Low tertile (score $0-3$; light gray lines) corresponds to lower adherence to $\mathrm{MeDi}$, middle tertile (score 4-5; dark gray lines) to middle adherence, and high tertile (score 6-9; black lines) to higher adherence. Figure is derived from a model that uses all subjects and is adjusted for cohort, age, sex, ethnicity, education, apolipoprotein E genotype, caloric intake, smoking, comorbidity index, and body mass index. Duration of follow-up is truncated at 10 years.

\section{Discussion}

This study suggests that higher adherence to the MeDi is associated with a reduction in risk for $\mathrm{AD}$ and slower cognitive decline. The gradual reduction in AD risk for higher tertiles of $\mathrm{MeDi}$ adherence also suggests a possible dose-response effect. The associations between $\mathrm{MeDi}$ and incident $\mathrm{AD}$ remained unchanged and significant even when simultaneously adjusting for the most commonly considered potential confounders for $\mathrm{AD}$, such as age, sex, ethnicity, education, APOE genotype, caloric intake, and body mass index. Higher adherence to $\mathrm{MeDi}$ reduced risk for probable $\mathrm{AD}$ either with or without coexisting stroke.

The association between high adherence to the $\mathrm{MeDi}$ and lower risk for $\mathrm{AD}$ may be mediated by the composite effect of some of its beneficial components, such as higher intake of fish, ${ }^{12,13}$ fruits, and vegetables rich in antioxidants such as vitamin $\mathrm{C}^{4-6}$ vitamin $\mathrm{E},{ }^{4-8}$ and flavonoids ${ }^{9}$ and higher intake of unsaturated fatty acids. ${ }^{10-12}$ Some of the inconsistencies regarding some of the above dietary elements and risk for $\mathrm{AD}$ in the existing literature may be a result of failure to consider possible additive and interactive (antagonistic or synergistic) effects among nutritional components, which may be captured in a composite dietary pattern such as the MeDi. For example, the effect of fish con- sumption in reducing blood pressure ${ }^{57}$ and blood lip$\mathrm{ids}^{58}$ appears to be much more pronounced in subjects following a low-fat diet. When individual components used to derive the MeDi pattern were examined in our study, mild-to-moderate alcohol consumption and higher vegetable intake were associated with decreased risk for $\mathrm{AD}$ in unadjusted models. Nevertheless, none of the individual components was a significant $\mathrm{AD}$ predictor when other confounders were considered. These results strengthen even further our initial hypothesis that composite dietary patterns can capture dimensions of nutrition that may be missed by individual components, and that an overall dietary pattern is likely to have a greater effect on health than a single nutrient.

The MeDi may play a role in multiple potential mechanisms including oxidative stress and inflammation, which are both important in the pathogenesis of AD. ${ }^{59}$ Complex phenols and many other substances with important antioxidant properties such as vitamin C, vitamin E, and carotenoid ${ }^{60-62}$ are found in high concentrations in the typical components of the MeDi. In the Attica epidemiological study, participants on the highest tertile of the MeDi score had 20\% lower CRP levels and 17\% lower interleukin-6 serum levels. ${ }^{63}$ Higher adherence to the $\mathrm{MeDi}$ also has been associated with significant reduction in various other inflammatory and coagulation markers including white blood cell counts and fibrinogen levels. ${ }^{63}$

Given the growing evidence for contribution of vascular risk factors in $\mathrm{AD}$ risk ${ }^{64,65}$ vascular mechanisms are important to consider. ${ }^{59}$ There is strong evidence relating the $\mathrm{MeDi}$ to lower risk for vascular risk factors such as hypertension, dyslipidemia, and diabetes. ${ }^{29,34,35,66}$ Subjects in the highest tertile of $\mathrm{MeDi}$ adherence have also been reported to have $15 \%$ lower homocysteine levels. ${ }^{63}$ Thus, vascular variables are likely to be in the causal pathway between $\mathrm{MeDi}$ and $\mathrm{AD}$ and should be considered as possible mediators. Because mediators, as contrasted with confounders, should not be controlled for in the statistical analysis, ${ }^{67}$ and in accordance with previous MeDi-related analytical approaches, ${ }^{68}$ we did not include specific vascular variables in our models.

Although most studies of the MeDi have been conducted in Mediterranean populations, recent studies have indicated MeDi-related health benefits in other populations such as northern European, ${ }^{68,69}$ Indian, ${ }^{35}$ and Australian populations, ${ }^{70}$ suggesting that the advantages of the $\mathrm{MeDi}$ are transferable to other populations. Our study provided the opportunity to examine the effect of MeDi in a multiethnic community in the United States, and our results support the notion that the beneficial effects of the $\mathrm{MeDi}$ are generalizable to different populations.

This study has limitations. The use of an a priori distribution-derived $\mathrm{MeDi}$ score assumes underlying 
monotonic effects, does not address possible thresholds or the shape of the underlying curve, and weighs equally the underlying individual food categories, which, in turn, are composed of different numbers of food constituents. Frequencies of food intake are based on relatively few diet constituents, which may underestimate the overall quantity of food in each food category, and a common limitation of studies of diet and disease is misclassification of exposure due to limited accuracy. However, assuming that the measurement error was random, our results may actually underestimate the association between high $\mathrm{MeDi}$ adherence and lower $\mathrm{AD}$ risk.

Despite the use of standard criteria, the diagnostic expertise of our center, and the thorough workup, there is always the possibility of disease misclassification bias. ${ }^{71}$ We partially addressed this issue by conducting secondary analyses considering only probable $\mathrm{AD}$ without stroke as the outcome, and our results were virtually unchanged. Subjects who were lost to follow-up were younger, less educated, and had more medical comorbidities, but $\mathrm{MeDi}$ was not related to age, education, or medical comorbidity burden. Also, the $\mathrm{MeDi}$ scores did not differ between subjects who remained in the study and those lost to follow-up. Therefore, it does not appear likely that our results could be explained by biases related to loss to followup.

It is possible that diet is related to socioeconomic status or to other habits or characteristics related to better health and a lower risk for $\mathrm{AD}$. In our data, $\mathrm{MeDi}$ was not related to education or the overall burden of medical comorbidities, but was related to ethnicity and smoking. We addressed this by adjusting for years of education, ethnic group, medical comorbidity index, and smoking, but we cannot completely rule out residual confounding as an explanation for our findings. Another confounder usually considered in nutritional epidemiology is caloric intake, ${ }^{42,72}$ and higher $\mathrm{MeDi}$ adherence was related to lower caloric intake in our data. We used caloric intake adjusted residuals for MeDi calculation and also included caloric intake as a covariate (as Willet and Stampfer ${ }^{42}$ recommended). We found that higher MeDi adherence was related to a lower risk for $\mathrm{AD}$ over and above caloric intake.

Despite an average follow-up of 4 years, several studies have shown that subtle cognitive changes antedate the clinical diagnosis of AD by many years. ${ }^{55,73}$ Therefore, it is still possible that lower adherence to the MeDi could represent a consequence and not a cause of $\mathrm{AD}$. We addressed this possibility in several ways. We found remarkable stability in $\mathrm{MeDi}$ scores over intervals greater than 7 years for subjects with multiple dietary assessments regardless of dementia status. In addition, the associations between MeDi score and incident $\mathrm{AD}$ did not change when subjects with mild cog- nitive deficits at baseline and subjects with less than 2 years of follow-up were excluded. Thus, bias due to preclinical disease does not appear to be a likely explanation for our findings.

Confidence in our findings is also strengthened by the following factors. Dietary data were collected with a previously validated instrument that was used widely in epidemiological studies. ${ }^{41} \mathrm{We}$ used an a priori developed dietary pattern. ${ }^{27,31,74}$ Measures for multiple potential $\mathrm{AD}$ risk factors have been recorded carefully and adjusted for in the analyses. The diagnosis of $\mathrm{AD}$ took place in a university hospital with expertise in dementia and was based on comprehensive assessment and standard research criteria. The patients were followed prospectively at relatively short intervals. The study is community-based and the population is multiethnic, increasing the external validity of the findings.

This study was supported by the NIH (National Institute on Aging, AG07232, M-X.T., R.M., J.A.L., AG07702, M-X.T., R.M., AG15294-06, J.A.L., 1K08AG20856-01, J.A.L.; RR00645, Columbia University General Clinical Research Center), the Charles S. Robertson Memorial Gift for Research in Alzheimer's disease (R.M), the Blanchette Hooker Rockefeller Foundation (R.M), the New York City Council Speaker's Fund for Public Health Research (J.A.L.), and the Taub Institute for Research on Alzheimer's Disease and the Aging Brain. N.S., R.M., J.A.L.).

\section{References}

1. Solfrizzi V, Panza F, Capurso A. The role of diet in cognitive decline. J Neural Transm 2003;110:95-110.

2. Luchsinger JA, Mayeux R. Dietary factors and Alzheimer's disease. Lancet Neurol 2004;3:579-587.

3. Mattson MP. Emerging neuroprotective strategies for Alzheimer's disease: dietary restriction, telomerase activation, and stem cell therapy. Exp Gerontol 2000;35:489-502.

4. Zandi PP, Anthony JC, Khachaturian AS, et al. Reduced risk of alzheimer disease in users of antioxidant vitamin supplements: the Cache County Study. Arch Neurol 2004;61:82-88.

5. Engelhart MJ, Geerlings MI, Ruitenberg A, et al. Dietary intake of antioxidants and risk of Alzheimer disease. JAMA 2002; 287:3223-3229.

6. Masaki KH, Losonczy KG, Izmirlian G, et al. Association of vitamin $\mathrm{E}$ and $\mathrm{C}$ supplement use with cognitive function and dementia in elderly men. Neurology 2000;54:1265-1272.

7. Morris MC, Evans DA, Bienias JL, et al. Vitamin E and cognitive decline in older persons. Arch Neurol 2002;59: $1125-1132$.

8. Morris MC, Evans DA, Bienias JL, et al. Dietary intake of antioxidant nutrients and the risk of incident Alzheimer disease in a biracial community study. JAMA 2002;287:3230-3237.

9. Commenges D, Scotet V, Renaud S, et al. Intake of flavonoids and risk of dementia. Eur J Epidemiol 2000;16:357-363.

10. Kalmijn S, Feskens EJ, Launer LJ, Kromhout D. Polyunsaturated fatty acids, antioxidants, and cognitive function in very old men. Am J Epidemiol 1997;145:33-41.

11. Morris MC, Evans DA, Bienias JL, et al. Dietary fats and the risk of incident Alzheimer disease. Arch Neurol 2003;60: 194-200.

12. Morris MC, Evans DA, Bienias JL, et al. Consumption of fish and n-3 fatty acids and risk of incident Alzheimer disease. Arch Neurol 2003;60:940-946. 
13. Barberger-Gateau P, Letenneur L, Deschamps V, et al. Fish, meat, and risk of dementia: cohort study. BMJ 2002;325: 932-933.

14. La Rue A, Koehler KM, Wayne SJ, et al. Nutritional status and cognitive functioning in a normally aging sample: a 6-y reassessment. Am J Clin Nutr 1997;65:20-29.

15. Clarke R, Smith AD, Jobst KA, et al. Folate, vitamin B12, and serum total homocysteine levels in confirmed Alzheimer disease. Arch Neurol 1998;55:1449-1455.

16. Wang HX, Wahlin A, Basun $\mathrm{H}$, et al. Vitamin B(12) and folate in relation to the development of Alzheimer's disease. Neurology 2001;56:1188-1194.

17. Ruitenberg A, van Swieten JC, Witteman JC, et al. Alcohol consumption and risk of dementia: the Rotterdam Study. Lancet 2002;359:281-286.

18. Mukamal KJ, Kuller LH, Fitzpatrick AL, et al. Prospective study of alcohol consumption and risk of dementia in older adults. JAMA 2003;289:1405-1413.

19. Luchsinger JA, Tang MX, Siddiqui M, et al. Alcohol intake and risk of dementia. J Am Geriatr Soc 2004;52:540-546.

20. Truelsen T, Thudium D, Gronbaek M, Copenhagen City Heart Study. Amount and type of alcohol and risk of dementia: the Copenhagen City Heart Study. Neurology 2002;59: 1313-1319.

21. Orgogozo JM, Dartigues JF, Lafont $S$, et al. Wine consumption and dementia in the elderly: a prospective community study in the Bordeaux area. Rev Neurol (Paris) 1997;153:185-192.

22. Kalmijn S, Launer LJ, Ott A, et al. Dietary fat intake and the risk of incident dementia in the Rotterdam Study. Ann Neurol 1997;42:776-782.

23. Luchsinger JA, Tang MX, Shea S, Mayeux R. Caloric intake and the risk of Alzheimer disease. Arch Neurol 2002;59: $1258-1263$.

24. Luchsinger JA, Tang MX, Shea S, Mayeux R. Antioxidant vitamin intake and risk of Alzheimer disease. Arch Neurol 2003; 60:203-208.

25. Engelhart MJ, Geerlings MI, Ruitenberg A, et al. Diet and risk of dementia: does fat matter?: the Rotterdam Study. Neurology 2002;59:1915-1921.

26. Crystal HA, Ortof E, Frishman WH, et al. Serum vitamin B12 levels and incidence of dementia in a healthy elderly population: a report from the Bronx Longitudinal Aging Study. J Am Geriatr Soc 1994;42:933-936.

27. Jacques PF, Tucker KL. Are dietary patterns useful for understanding the role of diet in chronic disease? Am J Clin Nutr 2001;73:1-2.

28. Lagiou P, Trichopoulou A, Henderickx HK, et al. Household budget survey nutritional data in relation to mortality from coronary heart disease, colorectal cancer and female breast cancer in European countries. DAFNE I and II projects of the European Commission. Data Food Networking. Eur J Clin Nutr 1999;53:328-332.

29. Panagiotakos DB, Pitsavos CH, Chrysohoou C, et al. Status and management of hypertension in Greece: role of the adoption of a Mediterranean diet: the Attica study. J Hypertens 2003;21:1483-1489.

30. Trichopoulou A, Kouris-Blazos A, Wahlqvist ML, et al. Diet and overall survival in elderly people. BMJ 1995;311: $1457-1460$.

31. Trichopoulou A, Costacou T, Bamia C, Trichopoulos D. Adherence to a Mediterranean diet and survival in a Greek population. N Engl J Med 2003;348:2599-2608.

32. Lasheras C, Fernandez S, Patterson AM. Mediterranean diet and age with respect to overall survival in institutionalized, nonsmoking elderly people. Am J Clin Nutr 2000;71:987-992.
33. Trichopoulou A, Lagiou P, Kuper H, Trichopoulos D. Cancer and mediterranean dietary traditions. Cancer Epidemiol Biomarkers Prev 2000;9:869-873.

34. de Lorgeril M, Salen P, Martin JL, et al. Mediterranean diet, traditional risk factors, and the rate of cardiovascular complications after myocardial infarction: final report of the Lyon Diet Heart Study. Circulation 1999;99:779-785.

35. Singh RB, Dubnov G, Niaz MA, et al. Effect of an IndoMediterranean diet on progression of coronary artery disease in high risk patients (Indo-Mediterranean Diet Heart Study): a randomised single-blind trial. Lancet 2002;360:1455-1461.

36. Luchsinger JA, Tang MX, Siddiqui M, et al. Alcohol intake and risk of dementia. J Am Geriatr Soc 2004;52:540-546.

37. Scarmeas N, Levy G, Tang MX, et al. Influence of leisure activity on the incidence of Alzheimer's disease. Neurology 2001; 57:2236-2242.

38. Hughes CP, Berg L, Danziger WL, et al. A new clinical scale for the staging of dementia. Br J Psychiatry 1982;140:566-572.

39. Stern Y, Andrews H, Pittman J, et al. Diagnosis of dementia in a heterogeneous population. Development of a neuropsychological paradigm-based diagnosis of dementia and quantified correction for the effects of education. Arch Neurol 1992;49: 453-460.

40. McKhann G, Drachman D, Folstein M, et al. Clinical diagnosis of Alzheimer's disease: report of the NINCDS-ADRDA Work Group under the auspices of Department of Health and Human Services Task Force on Alzheimer's Disease. Neurology 1984;34:939-944.

41. Willett WC, Sampson L, Stampfer MJ, et al. Reproducibility and validity of a semiquantitative food frequency questionnaire. Am J Epidemiol 1985;122:51-65.

42. Willett W, Stampfer M. Implications of total energy intake for epidemiological analyses. In: Willett W, ed. Nutritional epidemiology. New York: Oxford University Press, 1998:273-301.

43. Kuczmarski R, Carroll M, Flegal K, Troiano R. Varying body mass index cutoff points to describe overweight prevalence among U.S. adults: NHANES III (1988 to 1994). Obes Res 1997;5:542-548.

44. Census of Population and Housing. Summary tape file 1, technical documentation. Washington, DC: Bureau of the Consensus, 1991.

45. Scarmeas N, Brandt J, Albert M, et al. Delusions and hallucinations are associated with worse outcome in Alzheimer disease. Arch Neurol 2005;62:1601-1608.

46. Scarmeas N, Albert M, Brandt J, et al. Motor signs predict poor outcomes in Alzheimer disease. Neurology 2005;64: $1696-1703$.

47. Charlson ME, Pompei P, Ales KL, MacKenzie CR. A new method of classifying prognostic comorbidity in longitudinal studies: development and validation. J Chronic Dis 1987;40: 373-383.

48. Buschke H, Fuld PA. Evaluating storage, retention, and retrieval in disordered memory and learning. Neurology 1974;24: 1019-1025.

49. Benton A. The Visual Retention Test. New York: The Psychological Corporation, 1955.

50. Wechsler D. Wechsler Adult Intelligence Scale Revised. New York: The Psychological Corporation, 1981.

51. Mattis S. Mental status examination for organic mental syndrome in the elderly patient. In: Bellak L, Karasu TB, eds. Geriatric psychiatry. New York: Grune \& Stratton, 1976:77-121.

52. Rosen WG. The Rosen Drawing Test. Bronx, NY: Veterans Administration Medical Center, 1981.

53. Goodglass H, Kaplan D. The assessment of aphasia and related disorders. 2nd ed. Philadelphia: Lea \& Febiger, 1983. 
54. Benton AL, Hamsher K, Sivan AB. Manual for the multilingual aphasia examination. 3rd ed. Iowa City, IA: AJA Associates, 1994.

55. Scarmeas N, Albert SM, Manly JJ, Stern Y. Education and rates of cognitive decline in incident Alzheimer's disease. J Neurol Neurosurg Psychiatry 2006;77:308-316.

56. Stewart R, Masaki K, Xue Q-L, et al. A 32-year prospective study of change in body weight and incident dementia: the Honolulu-Asia Aging Study. Arch Neurol 2005;62:55-60.

57. Vandongen R, Mori TA, Burke V, et al. Effects on blood pressure of omega 3 fats in subjects at increased risk of cardiovascular disease. Hypertension 1993;22:371-379.

58. Mori TA, Vandongen R, Beilin LJ, et al. Effects of varying dietary fat, fish, and fish oils on blood lipids in a randomized controlled trial in men at risk of heart disease. Am J Clin Nutr 1994;59:1060-1068.

59. Cummings JL. Alzheimer's disease. N Engl J Med 2004;351: $56-67$.

60. Joshipura KJ, Hu FB, Manson JE, et al. The effect of fruit and vegetable intake on risk for coronary heart disease. Ann Intern Med 2001;134:1106-1114

61. Witztum JL. The oxidation hypothesis of atherosclerosis. Lancet 1994;344:793-795.

62. Byers T, Perry G. Dietary carotenes, vitamin C, and vitamin E as protective antioxidants in human cancers. Annu Rev Nutr 1992;12:139-159.

63. Chrysohoou C, Panagiotakos DB, Pitsavos C, et al. Adherence to the Mediterranean diet attenuates inflammation and coagulation process in healthy adults: the ATTICA Study. J Am Coll Cardiol 2004;44:152-158.

64. Breteler MM. Vascular risk factors for Alzheimer's disease: an epidemiologic perspective. Neurobiol Aging 2000;21:153-160.
65. Luchsinger J, Mayeux R. Cardiovascular risk factors and Alzheimer's disease. Curr Atheroscler Rep 2004;6:261-266.

66. Psaltopoulou T, Naska A, Orfanos P, et al. Olive oil, the Mediterranean diet, and arterial blood pressure: the Greek European Prospective Investigation into Cancer and Nutrition (EPIC) study. Am J Clin Nutr 2004;80:1012-1018.

67. MacMahon B, Trichopoulos D. Epidemiology: principles and methods. 2nd ed. Boston: Little Brown, 1996.

68. Trichopoulou A, Orfanos P, Norat T, et al. Modified Mediterranean diet and survival: EPIC-elderly prospective cohort study. Rapid responses. BMJ 2005;330:991.

69. Knoops KT, de Groot LC, Kromhout D, et al. Mediterranean diet, lifestyle factors, and 10-year mortality in elderly European men and women: the HALE project. JAMA 2004;292: 1433-1439.

70. Kouris-Blazos A, Gnardellis C, Wahlqvist ML, et al. Are the advantages of the Mediterranean diet transferable to other populations? A cohort study in Melbourne, Australia. Br J Nutr 1999;82:57-61.

71. Knopman DS, DeKosky ST, Cummings JL, et al. Practice parameter: diagnosis of dementia (an evidence-based review). Report of the Quality Standards Subcommittee of the American Academy of Neurology. Neurology 2001;56:1143-1153.

72. Willett W, Stampfer MJ. Total energy intake: implications for epidemiologic analyses. Am J Epidemiol 1986;124:17-27.

73. Elias MF, Beiser A, Wolf PA, et al. The preclinical phase of Alzheimer disease: a 22-year prospective study of the Framingham cohort. Arch Neurol 2000;57:808-813.

74. Trichopoulos D, Lagiou P. Mediterranean diet and cardiovascular epidemiology. Eur J Epidemiol 2004;19:7-8. 